\title{
Spin Behavior in the Rotating Reference Frame
}

\section{OVERVIEW}

We have seen that, at any given point in time, the interaction of a classical magnetic moment with an external magnetic field is equivalent to an instantaneous rotation of the moment about the field (UNIT B1.1). For a static field, the rotation is a constant precession about the field itself. In this unit, we wish to consider the effect of adding other magnetic fields. We are most interested in the combination of a radiofrequency (RF) field perpendicular to a much larger constant field.

The interest in the additional field stems from the need to generate a transverse component of the magnetization. The act of turning on a perpendicular field for some period of time should tip any magnetic moment, initially aligned along the original static field, away from that direction. Such rotations leave the classical moment precessing at an angle around the original static field $B$. (The more fundamental quantum picture allows only two spin states for any measurements along the static direction, either parallel or antiparallel. The use of a classical picture of a moment or spin precessing at some angle is still appropriate in MR.) Studying the effects of an added field is most easily done in a frame rotating at the Larmor frequency $\omega_{\mathrm{L}}=\gamma B$. Figure B1.2.1 shows a reference (primed) frame rotating clockwise around the $z=z^{\prime}$ axis relative to a laboratory (unprimed) frame. To obtain a field rotating at the Larmor (angular) frequency $\omega_{\mathrm{L}}=\gamma B$ for protons would require a frequency of $42.6 B \mathrm{MHz}$ with $B$ in Tesla. Current whole-body systems range from $0.2 \mathrm{~T}(8.52 \mathrm{MHz})$ to $8 \mathrm{~T}(340.8 \mathrm{MHz})$ with the most common high-field systems currently being $1.5 \mathrm{~T}(63.9 \mathrm{MHz})$ and $3 \mathrm{~T}(127.8 \mathrm{MHz})$.

Consider an RF field along the $x^{\prime}$ axis in the rotating frame. Such an RF field is called a left-circularly polarized field. The static (main) field is along the $z$ axis and the RF field is rotating around the main field. The total effective field observed in the rotating frame has three components. One is the main field, another one is the RF field, and the other one is a fictitious field generated by the rotation of the rotating frame. This field depends on how fast the RF field rotates. If the RF field has a frequency such that the fictitious field cancels the main field in the rotating frame, i.e., the RF field is the only field left in the rotating frame (see Fig. B1.2.1), then we call this "on-resonance." Under these circumstances, the spins develop a new angular frequency, given by $\vec{\omega}_{1}=-\gamma \vec{B}_{1}$ (the relevant Larmor expression in the rotating frame, see Figure B1.2.2).

For the on-resonance case, even very weak RF fields can be used to rotate a spin initially aligned along $\vec{B}$ into the plane transverse to the static field, or, for that matter, to any angle away from its initial alignment. The angle $\theta$ swept by the spin is equal to $\gamma B_{1} \tau$, where $B_{1}$ is the magnitude of the RF field and $\tau$ is the time duration of how long the tipping process applies. This is often called a $\theta$ pulse. As discussed above, the rotation will be most effective when the resonance condition $(\omega=\gamma B)$ is satisfied. Adding a static perpendicular field, on the other hand, to the original static field would not yield the same kind of rotation. According to the solutions of equation of motion of the magnetic moment worked out in UNIT B1.1 or the technical discussion in this Unit, it instead leads to a precession about the new, resultant field direction. When $\gamma B_{1} \tau$ is $90^{\circ}$, the magnetization will be tipped from $\hat{z}^{\prime}$ to $\hat{y}^{\prime}$ (see Figure B1.2.3A). If the fictitious field does not exactly cancel out the main field in the rotating frame, we call it "off-resonance" (see Figure B1.2.3C). In the laboratory frame, the spins will behave according to Figure B1.2.3B and Figure B1.2.3D for on-resonance and off-resonance conditions, respectively. 
The RF field described above is the so-called "transmit" field; it is produced by a coil system separate from the static field source. The precessing (or "excited") protons produce their own RF field at the Larmor frequency. This RF field is usually detected by an altogether different RF coil system referred to as the "receive" coil.

\section{TECHNICAL DISCUSSION}

\section{The Rotating Reference Frame}

Suppose we are making magnetic moment measurements in a reference frame rotating at the Larmor precession frequency $(\omega=\gamma B)$. Such a frame will rotate clockwise around the $z$ axis as seen from above the origin $(z>0)$ in a laboratory frame with a constant magnetic field pointing in the positive $z$ direction (see Figure B1.2.1). From this rotating perspective, the spin is no longer precessing about $\hat{z}$. This reference frame has proven to be very useful in describing MRI experiments, and a mathematical framework is given for it below.

In Figure B1.2.1, a laboratory (fixed) reference frame, denoted by the unprimed Cartesian coordinates $(x, y, z)$ and their associated unit vectors, is compared to another frame denoted by primed quantities $\left(x^{\prime}, y^{\prime}, z^{\prime}\right)$, which is rotating about the $z$ axis with respect to the fixed frame. The instantaneous rotation of this frame is defined by a rotational angular velocity vector $\vec{\Omega}$. The direction of this vector is the axis around which the primed frame is being rotated and its magnitude is the angular speed of rotation in radians per second. The angular velocity may be changing with time, $\vec{\Omega}=\vec{\Omega}(t)$.

We next consider some vector $\vec{V}$ in the laboratory frame. The time derivative of $\vec{V}$ in both frames are related by:

$$
\frac{d \vec{V}}{d t}=\left(\frac{d \vec{V}}{d t}\right)^{\prime}+\vec{\Omega} \times \vec{V}
$$

where the primed derivative is defined as:

Spin Behavior in the Rotating Reference Frame

\section{B1.2.2}

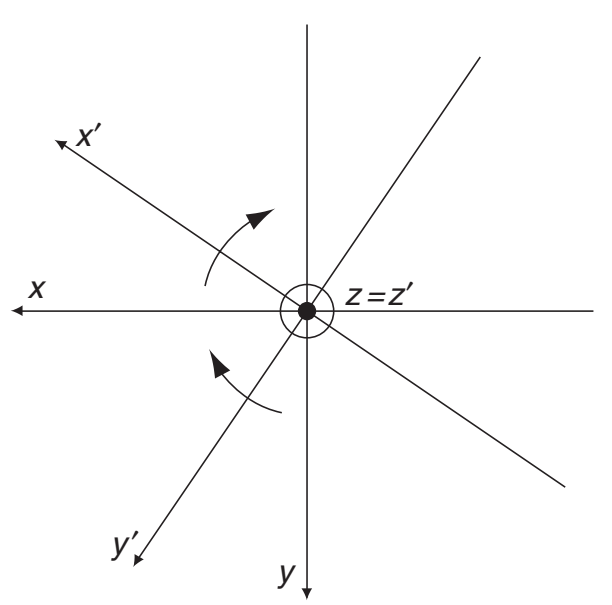

Figure B1.2.1 The sense of the rotation of a primed reference frame in which a magnetic moment is at rest. The primed frame rotates clockwise around the $z=z^{\prime}$ axis for a static magnetic field pointing in the $+\hat{z}$ direction according to a laboratory observer positioned above $(z>0)$ the $x-y$ plane. The rotation frequency of the primed reference frame is usually set to be the Larmor precession frequency. 


$$
\left(\frac{d \vec{V}}{d t}\right)^{\prime} \equiv \frac{d V_{x^{\prime}}(t)}{d t} \hat{x}^{\prime}(t)+\frac{d V_{y^{\prime}}(t)}{d t} \hat{y}^{\prime}(t)+\frac{d V_{z^{\prime}}(t)}{d t} \hat{z}^{\prime}(t)
$$

This derivative represents the rate of change of the vector quantity with respect to the rotating reference frame. The primed unit vectors represent the unit vectors in the rotating frame. (They are time dependent because they are rotating if an observer is sitting in the laboratory frame.) The primed subscript index of $V$ represents the component of vector $\vec{V}$ along that direction in the rotating frame.

Consider $\vec{\mu}$ as the vector quantity $\vec{V}(t)$ in Equation B1.2.1:

$$
\frac{d \vec{\mu}}{d t}=\left(\frac{d \vec{\mu}}{d t}\right)^{\prime}+\vec{\Omega} \times \vec{\mu}
$$

On the other hand, Equation B1.1.20, the equation of motion for the magnetic moment, is:

$$
\frac{d \vec{\mu}}{d t}=\gamma \vec{\mu} \times \vec{B}
$$

Using Equation B1.2.3 in Equation B1.2.4 yields the equation of motion in the rotating frame:

$$
\left(\frac{d \vec{\mu}}{d t}\right)^{\prime}=\gamma \vec{\mu} \times \vec{B}_{\text {eff }}
$$

The rotating frame acts to create an "effective magnetic field" given by:

$$
\vec{B}_{\text {eff }}=\vec{B}+\frac{\vec{\Omega}}{\gamma}
$$

This effective field is the superposition of the external magnetic field plus a fictitious magnetic field whose magnitude is $|\vec{\Omega}| / \gamma$ and whose direction is the same as that of the vector $\vec{\Omega}$. The rotational motion around $\vec{B}_{\text {eff }}$ is the by now familiar clockwise or left-handed precession (for an instant, at least) looking backwards along its direction.

The freedom to choose the primed frame is a key concept in the analysis of the magnetic moment behavior. The simplest case to consider is when $\vec{\Omega} / \gamma=-\vec{B}$. Since $(d \vec{\mu} / d t)^{\prime}$ is now zero from Equation B1.2.5, $\vec{\mu}$ must be a constant in the primed frame. The rotating frame simplifies our perspective of the motion of the spins.

\section{Creating Transverse Magnetization with an RF Field}

In order to detect the presence of any magnetization, it will be necessary to create a transverse component. We already understand that to rotate the magnetization, we must apply a magnetic field, say $\vec{B}_{1}$, perpendicular to the magnetization. Equation B1.2.5 implies that a field applied along $\hat{x}^{\prime}$ will cause $\vec{\mu}$ to rotate about $\hat{x}^{\prime}$, at least in the rotating 
frame. Practically, this means that $\vec{B}_{1}$ in the laboratory frame must be a time-varying field that acts like it is at rest in the rotating frame.

The usefulness of a rotating reference frame in the analysis of the static field is incentive to consider such frames for the combined static plus RF fields. The motivation can be made more compelling by the following picture: Imagine a spin precessing at a small angle around the static field direction. To tip this spin down to a larger angle, an additional field should be synchronized to push the spin down at a given position every time the spin comes back around in its precession to this same position.

A "left-circularly polarized" RF field is defined by:

$$
\vec{B}_{1}^{\mathrm{lcp}}(t)=B_{1}(\hat{x} \cos \omega t-\hat{y} \sin \omega t)=B_{1} \hat{x}^{\prime}
$$

It is obvious that this field is "at rest" in the rotating frame. Importantly, the (generally time-dependent) amplitude $B_{1}(t)$ is the full rotating frame amplitude available for spin flipping. The $90^{\circ}$ phase difference between the $x$ and $y$ coordinates of the laboratory field has led to calling Equation B1.2.7 a "quadrature" field. It takes two orthogonal coils to generate this field. Because of the power advantage, and other factors concerning signal-to-noise and the need for RF spatial homogeneity, such rotating fields are commonly used in MR.

\section{The Equation of Motion in Terms of the Effective Field}

The equation of motion (Equation B1.2.5) in the rotating reference frame for a spin immersed in the combination of the constant field $\vec{B}_{0}=B_{0} \hat{z}$ and the left-circularly polarized field (Equation B1.2.7) is:

$$
\begin{aligned}
\left(\frac{d \vec{\mu}}{d t}\right)^{\prime} & =\vec{\mu} \times\left[\hat{z}^{\prime}\left(\omega_{0}-\omega\right)+\hat{x}^{\prime} \omega_{1}\right] \\
& =\gamma \vec{\mu} \times \vec{B}_{\text {eff }}
\end{aligned}
$$

Because the rotating frame rotates around the $z$-axis, $\hat{z}^{\prime}=\hat{z}$. Appearing in Equation B1.2.8 are the Larmor frequency $\omega_{0} \equiv \gamma B_{0}$, the RF laboratory frequency $\omega$, and the spin-precession frequency $\omega_{1}$ associated with the circularly polarized RF field (Equation B1.2.7):

$$
\omega_{1} \equiv \gamma B_{1}
$$

The effective magnetic field is:

$$
\vec{B}_{\text {eff }} \equiv\left[\hat{z}^{\prime}\left(\omega_{0}-\omega\right)+\hat{x}^{\prime} \omega_{1}\right] / \gamma
$$

Spin Behavior in the Rotating Reference Frame

B1.2.4 


$$
\omega=\omega_{0} \quad \text { (on-resonance condition) }
$$

This choice represents the origin of the "resonance" reference in the acronyms NMR and MRI. Under this condition, the $B_{1}$ field is maximally synchronized to tip the spin around the $x^{\prime}$-axis. The first term is then eliminated in Equation B1.2.10, leading to the cornerstone equation of motion in the presence of an RF transmit field $B_{1}$ :

$$
\left(\frac{d \vec{\mu}}{d t}\right)^{\prime}=\omega_{1} \vec{\mu} \times \hat{x}^{\prime} \quad\left(\text { when } \omega=\omega_{0}\right)
$$

There is then only a precession about the $\hat{x}^{\prime}$ axis with the precessional frequency $\omega_{1}$ given in Equation B1.2.12.

\section{The RF Precession in More Detail}

The fact that the magnetic resonance effect is more easily understood in the appropriate rotating reference frame is also reflected by the manner in which an analytical solution is found for the motion of the magnetic moment. In the rotating frame, let the RF field be constant along $\hat{x}^{\prime}$, then:

$$
\vec{B}_{\text {eff }}=\vec{B}_{1}=B_{1} \hat{x}^{\prime}
$$

for the on-resonance case. The magnetic moment vector motion is found by transcribing the solution to the Bloch equation given in Equation B1.1.31 according to the substitutions $z \rightarrow x^{\prime}, y \rightarrow z^{\prime}, x \rightarrow y^{\prime}$ or by directly solving Equation B1.2.12

$$
\begin{aligned}
& \mu_{x^{\prime}}(t)=\mu_{x^{\prime}}(0) \\
& \mu_{y^{\prime}}(t)=\mu_{y^{\prime}}(0) \cos \theta(t)+\mu_{z^{\prime}}(0) \sin \theta(t) \\
& \mu_{z^{\prime}}(t)=-\mu_{y^{\prime}}(0) \sin \theta(t)+\mu_{z^{\prime}}(0) \cos \theta(t)
\end{aligned}
$$

with

$$
\theta(t)=\omega_{1} t
$$

and $\omega_{1} \equiv \gamma B_{1}$. The general time dependent case (with arbitrary initial time $t_{0}$ ) is solved by the substitution $\vec{\mu}(0) \rightarrow \vec{\mu}\left(t_{0}\right)$ where:

$$
\theta(t)=\int_{t_{0}}^{t} d t^{\prime} \omega_{1}\left(t^{\prime}\right)
$$

and

$$
\omega_{1}(t)=\gamma B_{1}(t)
$$




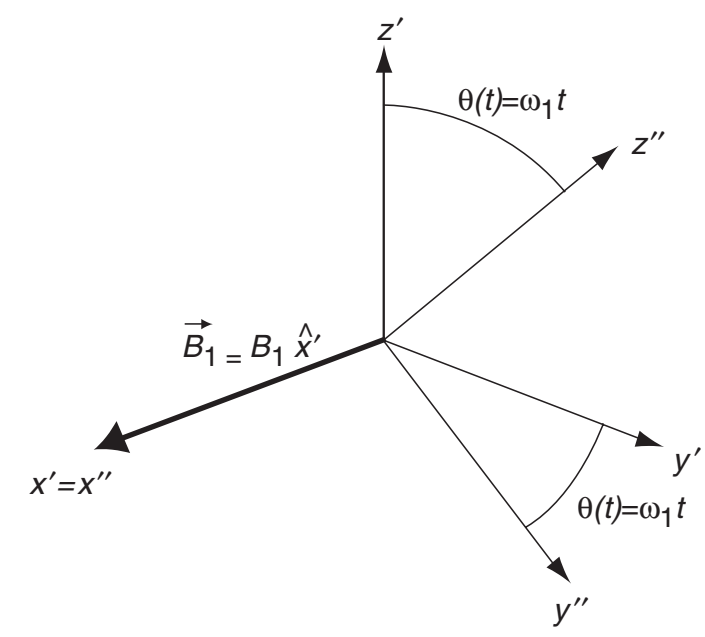

Figure B1.2.2 The double-primed reference frame rotating about the RF field, $\vec{B}_{1}$, which is along the $\hat{x}^{\prime}$ direction in the primed reference frame. The spin is initially along the $\hat{Z}^{\prime}$ direction and is subsequently tipped by an angle $\theta(t)$ by the $\vec{B}_{1}$ field. The $\hat{z}^{\prime \prime}$ axis remains aligned along the spin direction during the rotation.

When an RF pulse is applied for a finite time $t$, the spin (or the rotating frame) rotates an angle $\theta(t)$ (Figure B1.2.2). This RF pulse is referred to as a $\theta$-pulse. For example, if $\theta=$ $\pi / 2$, then the RF pulse is called a $\pi / 2$ pulse or a $90^{\circ}$ pulse.

\section{The Flip-Angle Formula and a Specific Illustration}

A $B_{1}$ field applied on-resonance for a finite time is called an "RF pulse". Suppose the RF field is turned on quickly to a constant value $B_{1} \hat{x}^{\prime}$ for a time interval $\tau$ and then it is just as rapidly turned off. From the precession lessons of UNITB1.1 (or from the explicit solutions of the previous subsection), Equation B1.2.12 implies that the spin rotates through the angle

$$
\theta=\gamma B_{1} \tau
$$

around $\hat{x}^{\prime}$. For example, the size of $B_{1}$ required for a $90^{\circ}$ flip angle over $1.0 \mathrm{msec}$ is 5.87 $\mu \mathrm{T}(0.0587 \mathrm{G})$ for protons.

An illustration of various spin trajectories is helpful in highlighting the effectiveness of being on-resonance for tipping spins. Trajectories in the primed and unprimed frames for both off-resonance and on-resonance conditions are shown in Figure B1.2.3. The pictures are generated from the solutions of the equation of motion for a constant field $B_{1} \hat{x}^{\prime}$ in the rotating frame. The pure on-resonance rotation about the $x^{\prime}$ axis is demonstrated in Figure B1.2.3A. The corresponding spiraling down found in the laboratory is shown in Figure B1.2.3B. For the example given above, if $B_{0}=1 \mathrm{~T}$, then the spin will rotate in the laboratory frame more than 40,000 times before it hits the $x-y$ plane. The off-resonance motion in the primed frame (Figure B1.2.3C) shows the expected precession about the total field. In Figure B1.2.3D, the laboratory picture is that of a superposition of this precession on top of the rotation of the primed frame. On-resonance, even a weak RF field would readily rotate the spin down into the transverse plane. By contrast, the farther off-resonance a

pin Behavior in the Rotating Reference Frame
B1.2.6 
A

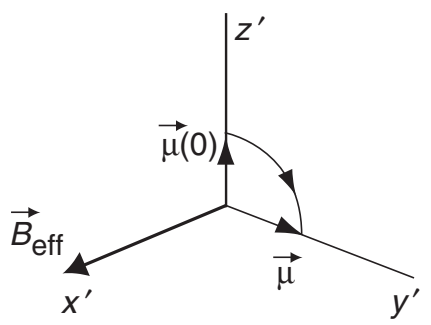

C

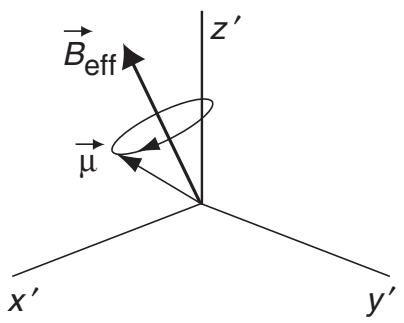

B

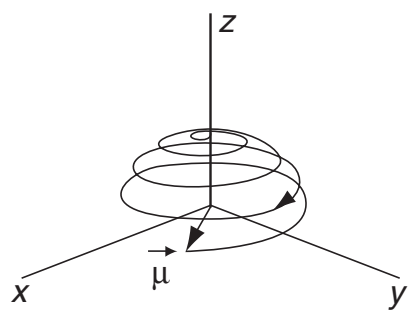

D

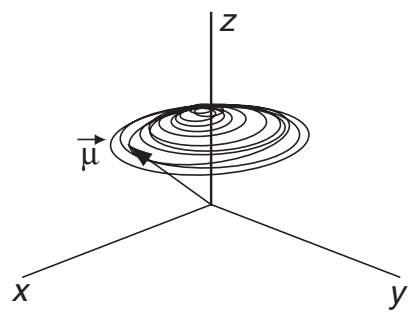

Figure B1.2.3 An on-resonance $\pi / 2$ spin flip as viewed in the rotating (A) and laboratory (B) frames for $\omega=\omega_{0}$ and $\omega_{1}=0.06 \omega_{0}$. An off-resonance trajectory as viewed in the primed (C) and unprimed (D) frames corresponds to the offset value, $\omega=0.85 \omega_{0}$, with $\omega_{1}=0.06 \omega_{0}$. In MR applications, the frequency $\omega_{1}$ would be much smaller in relation to the RF frequency, but the spiraling would then be much too dense to illustrate.

spin becomes, the closer the effective field is to the static field, and the less the spin is tipped from the vertical.

\section{KEY REFERENCES}

Goldstein, H. 1980. Classical Mechanics. Addison-Wesley, Reading, Mass.

This text reviews fundamental physics of classical mechanics.

Haacke, E.M., Brown, R.W., Thompson, M.R., and Venkatesan, R. 1999. Magnetic Resonance Imaging: Physical Principles and Sequence Design. John Wiley \& Sons, New York.

This text covers the technical aspects presented here, but in more detail, and also discusses more advanced materials.

Rabi, I.I., Ramsey, N.F., and Schwinger, J. 1954. Rotating coordinates in magnetic resonance problems. Rev. Modern Phys. 26:167.

This article presents an outstanding discussion of NMR in rotating reference frames.

Slichter, C.P. 1990. Principles of Magnetic Resonance, 3rd ed. Springer-Verlag, New York.

This text reviews the relevant general concepts of rotation and precession in classical mechanics and NMR.

Contributed by Yu-Chung Norman Cheng and E. Mark Haacke

Case Western Reserve University

Cleveland, Ohio

and The MRI Institute for Biomedical Research

St. Louis, Missouri 